\title{
Through-the-Thickness Compressive Strengths of Graphite/Epoxy Laminated Composites: Experimental Characterization and Statistical Analysis
}

\author{
Chao Zhang, R. Ganesan and Suong V. Hoa \\ Concordia Centre for Composites \\ Department of Mechanical Engineering \\ Concordia University \\ Montreal, Quebec, Canada H3G 1 M8
}

\begin{abstract}
The purpose of the present paper is to investigate the through-the-thickness compressive strengths of graphite/epoxy laminated composites. The investigation is focused on three aspects of concern; probabilistic characteristics, effects of specimen lay-ups and effects of specimen sizes involved with the compressive strengths. One type of cross-ply prism specimen and four types of unidirectional prism specimens with different sizes are tested by directly applying the through-the-thickness compressive load on the surfaces of specimens through two pieces of collars. The inherent variability in the through-the-thickness compressive strengths is statistically studied based on experimental results. A contact finite element analysis is performed to look into the effects of friction between the collar and specimen and the effects of modulus of collar materials on the stress distribution inside the specimen. It is found that the through-the-thickness compressive strength strongly depends on the specimen lay-up, and depends on the specimen size to some degree in the unidirectional specimens. Moreover, the through-the-thickness modulus also seems to depend on the specimen lay-ups. The probability function of the through-the-thickness compressive strength is given in order to demonstrate the involved random behavior.
\end{abstract}

\section{KEYWORDS}

through-the-thickness compressive strength, unidirectional and cross-ply prism specimens, matrix crack, rank-sum test, Kolmogorov-Smirnov goodness-of-fit test, Weibull distribution.

\section{INTRODUCTION}

Through-the-thickness (i.e., out-of-plane) compressive strengths of laminated composites are an essential material property when the failure of composite structures with $3 \mathrm{D}$ stress state is evaluated using the quadratic failure theory, i.e., Tsai-Wu or Hoffman criteria /1,2/. The 3D stress state usually exists in thin laminated composites with material discontinuities, geometry discontinuities and load-path eccentricities or in thick laminated composites. Furthermore, through-the-thickness compressive strength governed by the matrix materials could be used to evaluate the autoclave curing quality of thick laminated composites.

The through-the-thickness (abbreviated as TTH hereafter) tensile strength of unidirectional laminated composites has received a great deal of attention and a number of papers can be found $13-6 /$. However,

\footnotetext{
- Author to whom correspondence should be addressed.
} 
few papers are available which deal with the TTH compressive strengths; to the authors' knowledge, only Goeke $/ 7 /$ and Benzeggagh et al. /8/ performed the associated investigation. In the first paper, Goeke $/ 7 /$ reported the experimental studies on the compressive strengths of thick glass/polyester and glass/epoxy composites along the thickness and fiber directions in the U.S. Army Materials Technology Laboratory (MTL). The end-loaded prism specimens, cut from the thick composite plates, were used and the compressive load was directly imposed on two opposite surfaces of the specimen. Some important factors that would possibly affect the TTH compressive strengths, e.g., effects of collars, and specimen sizes, were carefully studied in the TTH compressive test. It was concluded by the MTL that the compressive test based on endloaded prism specimens is valid, because not only does the failure usually take place inside specimen gage section, but also the reproducibility of experimental data is also observed from specimen to specimen. In the second paper, Benzeggagh et al. /8/ experimentally measured the TTH compressive strengths of graphite/epoxy $\mathrm{T} 300 / 914$ and glass/epoxy M10 composites in order to evaluate the strength tensors in Tsai-Wu criterion in each case. The end-loaded prism specimens were also used with a $7 \mathrm{~mm} \times 7 \mathrm{~mm} \times 7 \mathrm{~mm}$ cubic shape. Because the concern of their paper is to study the Tsai-Wu strength tensors, detailed testing information and experimental results were not presented.

The intent of the present paper is to report the experimental and statistical investigation on the TTH compressive strengths of CYTEC $($ G40-800/5276-1 composite material, which is a part of the systematic investigation performed at the Concordia Centre for Composites $/ 9 \%$. The purpose of this investigation is to develop innovative delamination criteria towards laminated composites, in order that the effects of fiber orientations adjacent to the interfaces on delamination failure could be taken into consideration. The interlaminar shear strengths, and TTH tensile and compressive strengths are required as input data to the developed delamination criteria. Therefore, the TTH compressive strengths of laminated composites are experimentally characterized by the TTH compressive test based on the end-loaded prism specimens. The G40800/5276-1 composite material is selected as the candidate material because of its extensive application in aerospace structures. The experimental and statistical investigation is focused on three aspects of concern in the TTH compressive strength; probabilistic characteristics through a large number of specimens, effects of specimen lay-ups through unidirectional and cross-ply specimens, and effects of specimen sizes through four types of unidirectional specimens with different sizes. The inherent relationships between the TTH compressive strengths and the specimen failure modes are revealed by observing the directions of crack propagation. Also, the other material properties of G40800/5276-1 composite material dominated by the matrix material, such as in-plane transverse tensile and shear, and interlaminar shear strengths, are presented so as to provide a comparison between the TTH compressive strengths and other material properties.

\section{MATRIX-DOMINATED MATERIAL PROPERTIES}

The TTH compressive strength is a matrixdominated material property, like interlaminar and inplane shear strengths. Therefore, statistical behaviors of the other matrix-dominated strengths may shed some light into the TTH compressive strength. The experimental results for transverse tensile strength $Y_{T}$, in-plane shear strength $S_{12}$, and out-of-plane shear strengths $S_{13}$ and $S_{23}$ of G40-800/5276-1 composite material are thus demonstrated in this section. The experimental results are obtained from the unidirectional specimens with the sample size equal to 35 in each case /9/. The transverse tensile test is based on the ASTM Standard Test Method for Tensile Properties of Fiber-Resin Composites (D 3039) in which the specimens have straight-sides and constant crosssection with bonded tabs for load introduction. The inplane shear test is based on the ASTM Standard Guide for Testing In-plane Shear Properties of Composite Laminates (D 4255) in which the rail shear is applied to rectangular specimens through three rails. The out-ofplane shear test is based on the modified double notch 
Table 1

Experimental results of matrix-dominated strengths for G40-800/5276-1 composite material/9/

\begin{tabular}{|c|c|c|c|c|}
\hline & $\mathrm{Y}_{\mathrm{T}}$ & $\mathrm{S}_{12}$ & $\mathrm{~S}_{13}$ & $\mathrm{~S}_{23}$ \\
\hline$\mu(\mathrm{MPa})$ & 41.21 & 35.24 & 44.75 & 61.68 \\
\hline $\mathrm{CV}(\%)$ & 11.58 & 15.08 & 20.73 & 9.76 \\
\hline
\end{tabular}

$\mathrm{Y}_{\mathrm{T}}$ - transverse tensile strength

$\mathrm{S}_{12}-$ in-plane shear strength

$S_{13}$ - longitudinal interlaminar shear strength

$\mathrm{S}_{23}-$ transverse interlaminar shear strength

shear test and detailed information is available in Ref. 10.

The mean values and coefficients of variation obtained from the 35 sample values in each case for the matrix-dominated strengths are tabulated in Table 1; large variations are observed for each strength component. This indicates that the matrix-dominated strengths have high degree of randomness due to uncertainty of matrix defects. Therefore, the sample probability distributions are also presented herein, which are calculated by

$$
F(\sigma)=\frac{(i-1)+0.7}{n+0.4}
$$

where $i$ is the ordered rank of sample values in an ascending consequence, $\sigma$ is the sample value corresponding to the $i$-th rank, and $n$ is the total number of sample values (herein $n=35$ ). Then, each strength component is viewed as one continuous random variable and assumed to have the two-parameter Weibull distribution:

$$
F(\sigma)=1-\exp \left[-(\sigma / \alpha)^{\beta}\right]
$$

In the above, $\alpha$ is called as scale parameter and $\beta$ as shape parameter. The scale parameter is roughly equal to the mean value of the random variable, and the shape parameter represents the degree of randomness, with a small value suggesting large scatter involved in the random variable. These two parameters could be generated using the goodness-of-fit of the Weibull function in Equation (2) to the sample probability distribution in Equation (1). The sample (denoted by dots) and Weibull (denoted by solid lines) probability distributions for the four strength components are displayed in Figures 1 to 4 . It can be observed that the strengths $S_{12}$ and $S_{13}$ have small values of shape parameters because of large scatter.

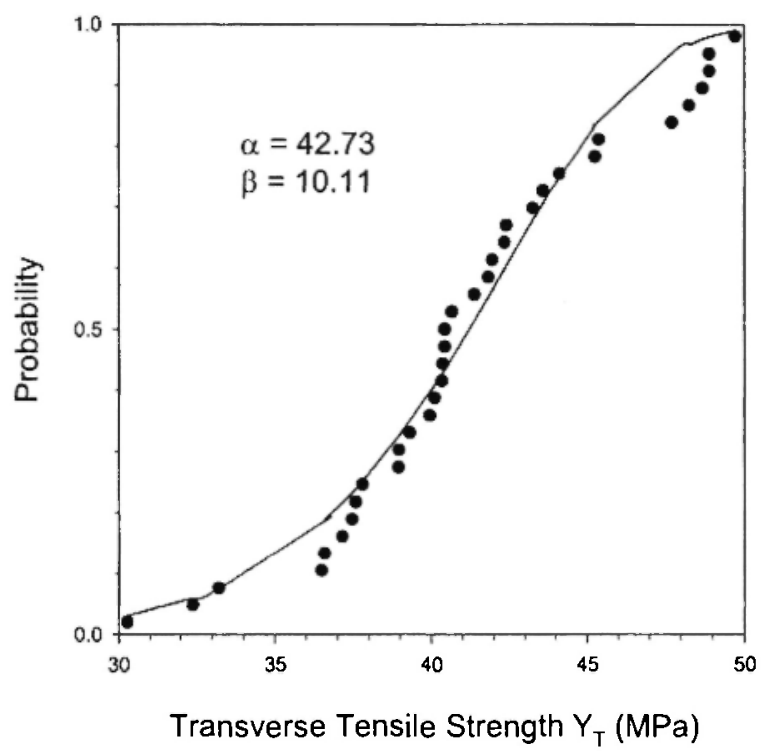

Fig. 1: Probability distribution of transverse tensile strength $Y_{T}$.

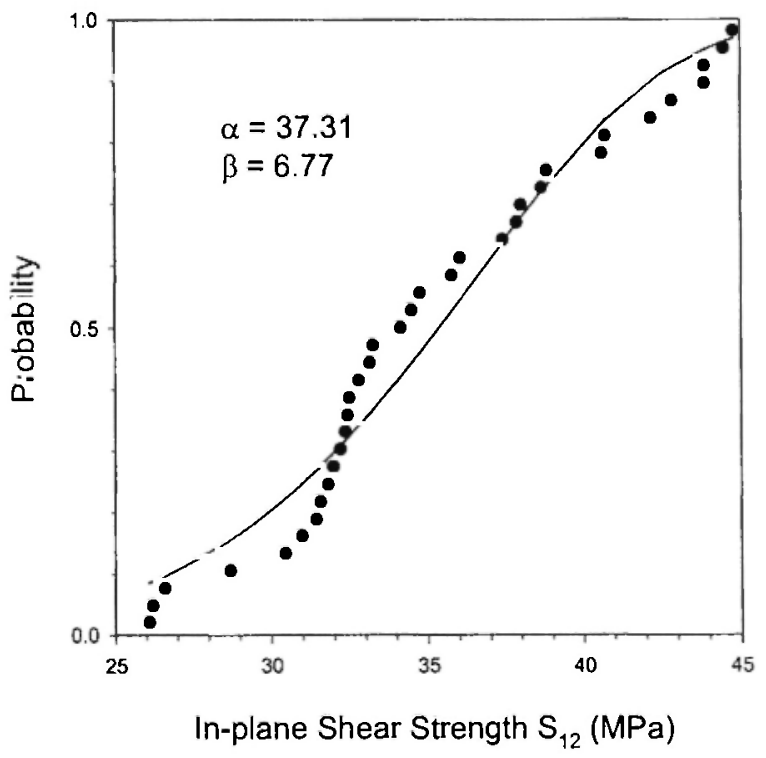

Fig. 2: Probability distribution of in-plane shear strength $\mathrm{S}_{12}$. 


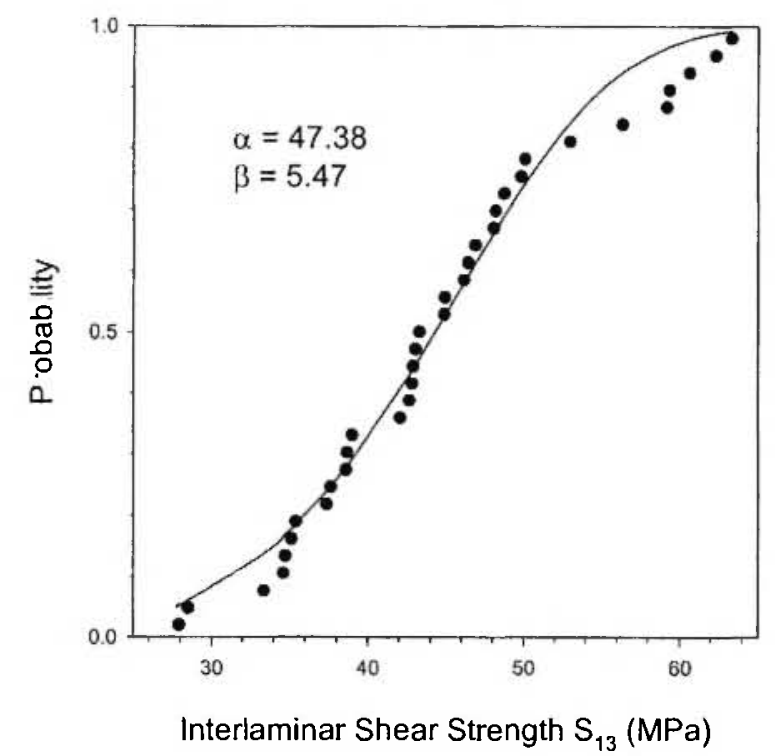

Fig. 3: Probability distribution of longitudinal interlaminar shear strength $\mathrm{S}_{13}$.

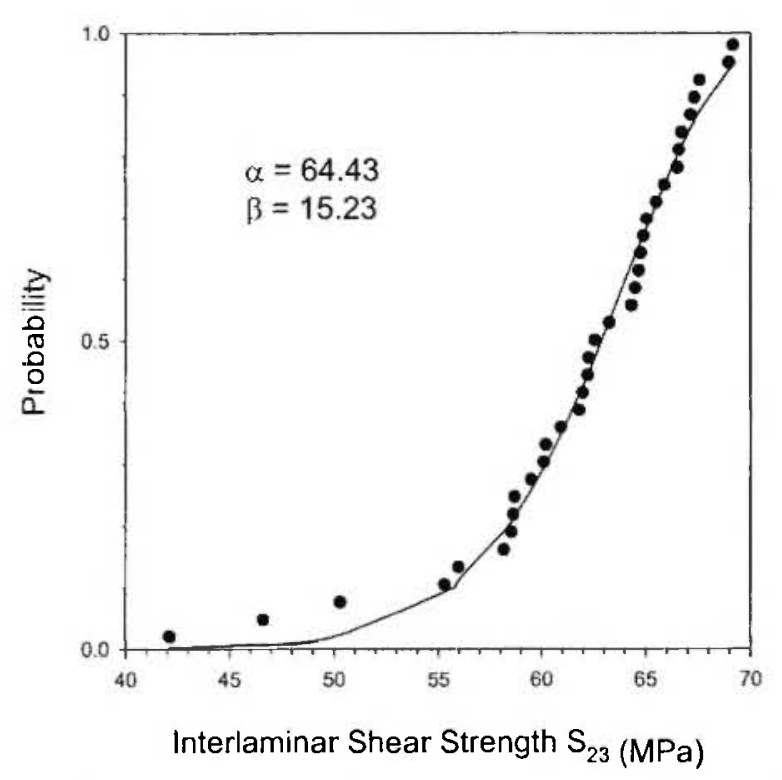

Fig. 4: Probability distribution of transverse interlaminar shear strength $S_{23}$.

\section{SPECIMEN DESIGN AND FINITE ELEMENT ANALYSIS}

The testing set-up employed in the present experimental program is schematically demonstrated in Figure 5. Thick prism specimens composed of 72 plies

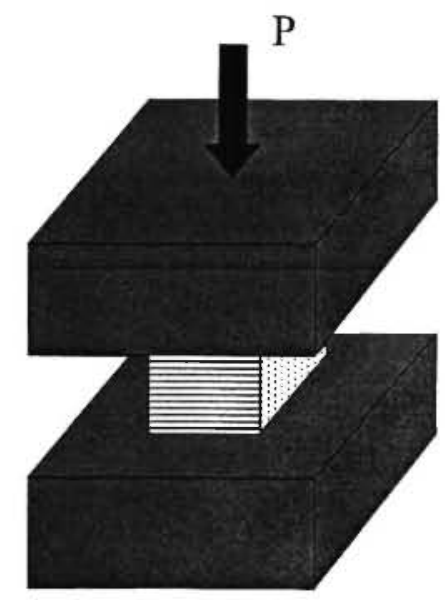

Fig. 5: The set-up for the TTH compressive test based on end-loaded prism specimens.

are used which have a nominal thickness of $13.32 \mathrm{~mm}$. The TTH compressive strengths are studied on $[0]_{72}$ unidirectional and $[0 / 90]_{36}$ cross-ply laminates respectively in order to uncover the effects of fiber orientations. Moreover, unidirectional specimens with different sizes, as shown in Figure 6, are also used to characterize the potential effects of specimen sizes on the TTH compressive strengths. The nominal specimen sizes (cross-section) and numbers are tabulated in Table 2. Considering that the TTH compressive strength of the cross-ply specimens is very high as shown in the following, the TTH compressive test is only performed on the cross-ply prism specimens with small crosssection due to the limitation of loading capacity of testing machine. Furthermore, instead of the aluminum collars for the unidirectional specimens, the carbon steel collars are used for cross-ply specimens because the TTH compressive strengths of the cross-ply laminates are even higher than the yield sirength of aluminum.

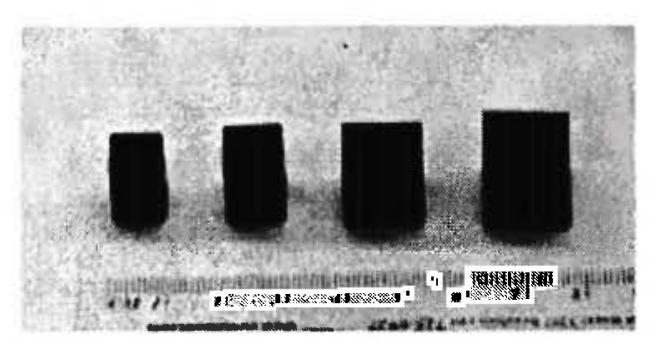

Fig. 6: Differently-sized prism unidirectional specimens. 
Table 2

Cross-sectional geometry and numbers of unidirectional and cross-play specimens

\begin{tabular}{|c|c|c|c|c|c|}
\hline & \multicolumn{4}{|c|}{ Unidirectional } & \multirow[t]{2}{*}{ Cross-Ply } \\
\hline & Group 1 & Group 2 & Group 3 & Group 4 & \\
\hline $\begin{array}{l}\text { Nominal Size } \\
(\mathrm{mm} \times \mathrm{mm})\end{array}$ & $8.5 \times 8.5$ & $10 \times 10$ & $12.7 \times 12.7$ & $15 \times 15$ & $8 \times 8$ \\
\hline $\begin{array}{c}\text { No. of } \\
\text { Specimens }\end{array}$ & 5 & 5 & 32 & 5 & 3 \\
\hline
\end{tabular}

All the specimens have a nominal thickness of $13.32 \mathrm{~mm}$.

Both the aluminum and carbon steel collars have a thickness of $12.7 \mathrm{~mm}$ and their cross-section areas are larger than those of specimens.

Technically, the experimental characterization of TTH compressive strengths of composite materials is not a difficult task, because the test technique is straightforward; i.e., to apply compressive loads to prism composite specimens along the thickness direction. The prism specimens can be conveniently made by cutting through the flat composite plates, while the compressive loads can be directly applied to the prism specimens by moving up the bottom plateau of testing machine without any need for loading introduction fixture. From the point of view of material failure evaluation, the experimental results used as input data to failure criteria should represent the material properties rather than the structural properties. The interaction between the prism specimens and the collars consists of a contact problem and therefore the coefficient of friction and the modulus of collar materials would affect the stress distribution inside the specimen. The material failure might be initiated at the contact interface rather than inside the specimen gage section as desired, and thus the actual material properties could not be derived from the experiments. Therefore, the effects of friction and collar modulus on the stress distribution need to be carefully investigated in order to reduce negative influence on the experimentally measured TTH compressive strengths. In the present paper, this investigation is achieved by a finite element analysis (FEA) using the ANSYS® FEA software.

Herein the finite element analysis is only performed on the unidirectional specimens together with the aluminum collars, and two cases are considered with the coefficients of friction $f=0.1$ and $f=0.3$. The interaction between the specimens and collars is modeled as a nonlinear surface-to-surface contact problem; the specimens and collars are meshed into 20 node brick elements, and the target surface attached to the collars is meshed into 8-node target elements and the contact surface attached to the specimens into 8node contact elements. Only one eighth of the system is employed for the finite element analysis due to the structural symmetry. The FEA model is illustrated in Figure 7, wherein the origin of the coordinate system is located at the specimen center and the $x$ axis corresponds to the fiber direction of unidirectional specimens. A surface load is applied to the aluminum collar with such a magnitude that generates a nominal TTH compressive stress of $200 \mathrm{MPa}$ inside the specimens. This magnitude of TTH compressive stress is close to the TTH compressive strength, as shown by the experimental results. The inherent purpose is to uncover the loading-dependent interaction between the unidirectional specimens and aluminum collars at the critical moment of specimen failure.

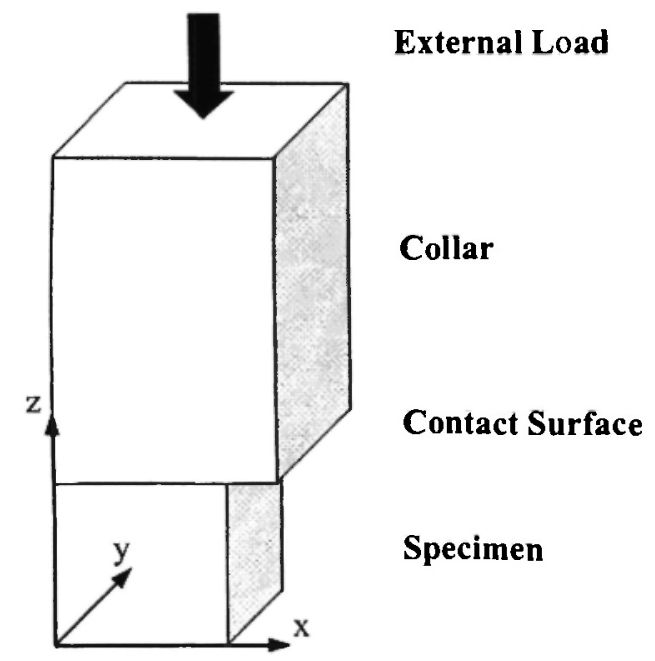

Fig. 7: Non-linear finite element model for the collarspecimen contact problem.

The FEA results for the three stress components are displayed in Figures 8 and 9, which would play an important role in the formation of matrix cracks. It can be seen that the maximum stress state for all the three stress components is generated near the specimen edges; the shear stress $\tau_{y z}$ along the entire edge parallel to the 


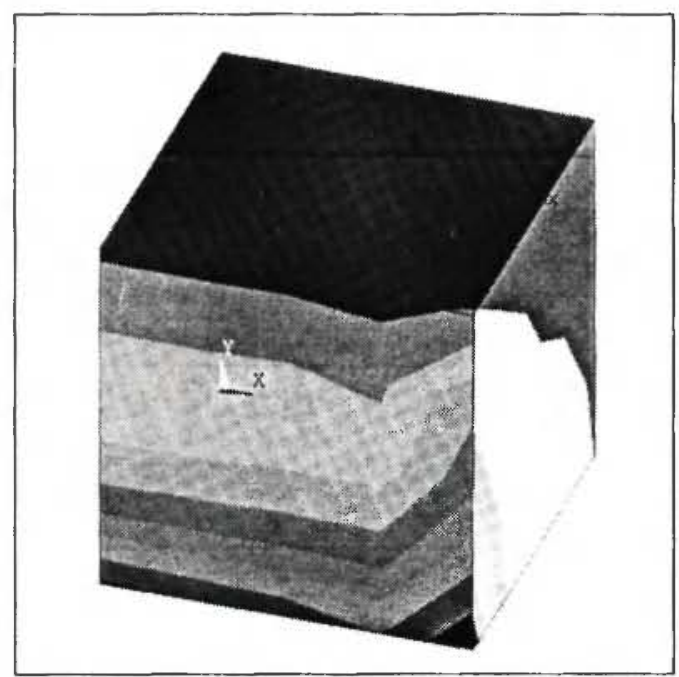

ANSYS 5.6

MAY 182000

$13: 17: 46$

NODAL SOLUTION

STEP $=1$
SUB $=6$

T IME $=100$

SY (AVG)

RSYS $=0$

PowerGraphies

EFACET $=1$

AVRES $=$ Mat

DMX $=.158622$

$S M N=-49.254$

SMX $=.59792$

-49.254
-43.715

$-38.176$

$-32.637$

$\square \begin{array}{r}-27.098 \\ -21.559\end{array}$

$-16.02$

ए] -10.48

$-4.941$

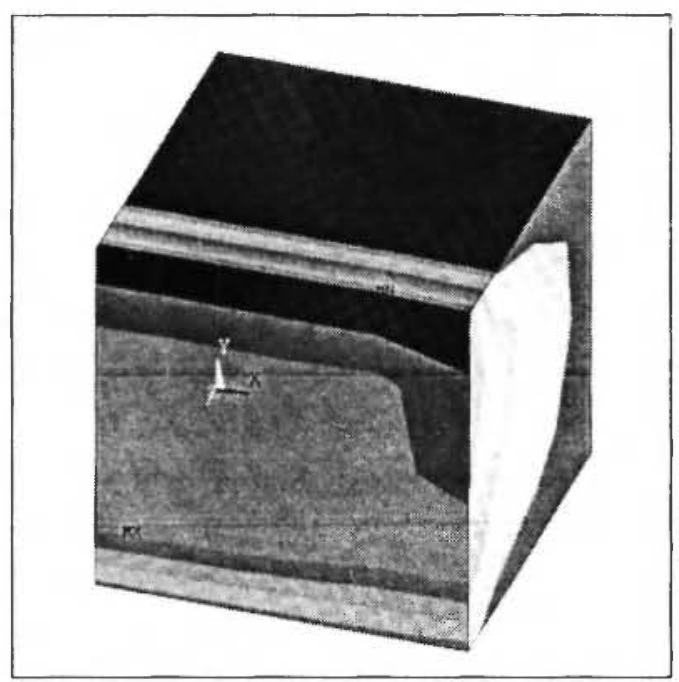

ANSYS 5.6

MAY 182000

$13: 20: 48$

NODAL SOLUTION

STE $P=1$

SUB $=6$

T IME $=100$

SYZ (AVG)

RSYS $=0$

Fowergraphics

EFACET $=1$

AVRES $=$ Mat

$\mathrm{DMX}=.158022$

SMN $=-20.678$

SMX $=.667967$

$-20.678$

$-18.306$

$-15.934$

$-13.562$

$-11.191$

$-8.819$

$-6.447$

$-4.075$

$-1.704$

.667967

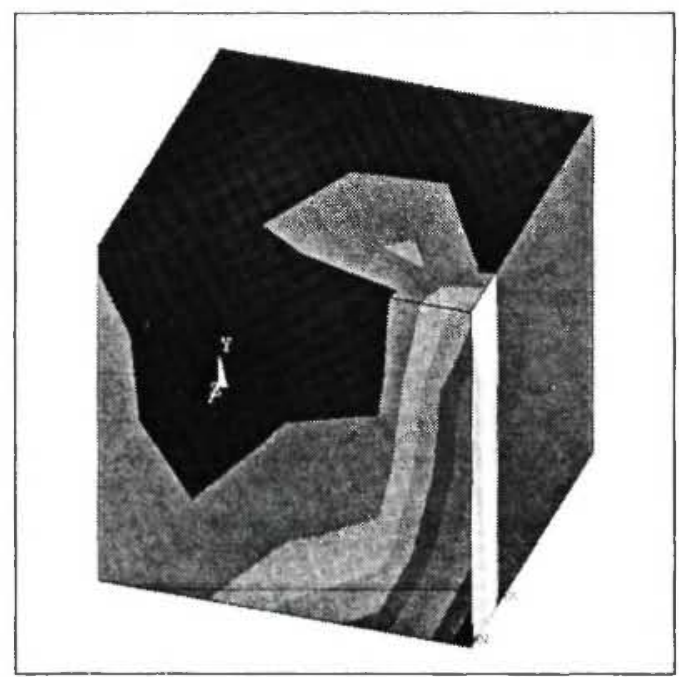

ANSYS 5.6

MAY 182000

13:19:15

NODAL SOLUTION

STEF $=1$

SUB $=6$

$T$ I ME $=100$

SXE

(AVG)

RSYS=0

PowerGraphics

EEACET $=1$

AVRES $=$ Mat

DMX $=.1580: 2$

$\mathrm{SMN}=-20.004$

$\mathrm{SMK}=.85421$

-20.004
-17.087

-17.057
-15.300

$-13.05$

$-10.73$

$\Rightarrow-8.416$

-6.009
-3.781

$-1.403$

.85421

Fig. 8: Distribution of stress components $\sigma_{y}, \tau_{y z}$ and $\tau_{x z}$ inside unidirectional specimens when $f=0.1$. 


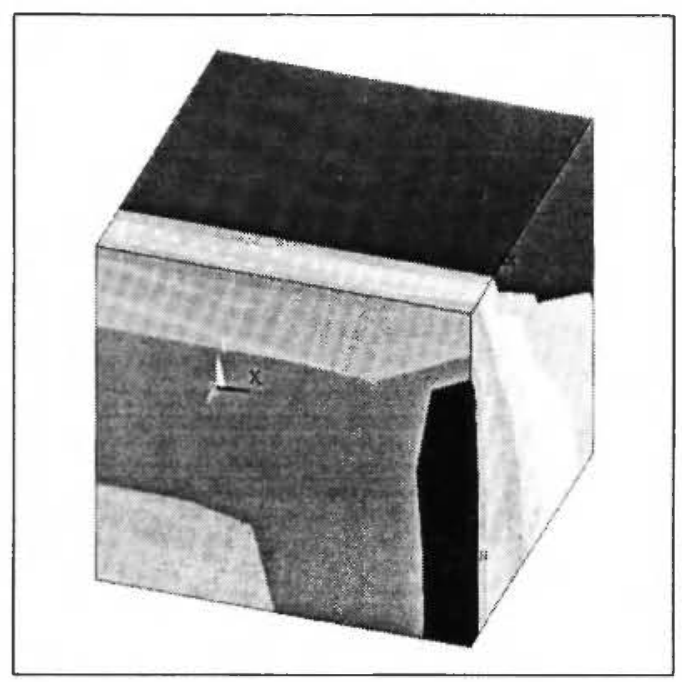

ANSYS 5.6

MAY 182000

$13: 42: 00$

NODAL SOLUTION

$S T E P=1$

UB $=7$

TIME $=100$

$S Y$

RSYS $=0$

(AVG)

PowerGraphics

EFACET $=1$

AVRES=Mat

DMX $=.150897$

SMN $=-64.533$

$\operatorname{SMX}=4.309$

$\begin{array}{r}-64.533 \\ -56.884 \\ \hline\end{array}$

$-49.235$

$-41.586$

$-33.937$

$-26.287$

$-18.638$

$-10.989$

$-3.34$

4.309

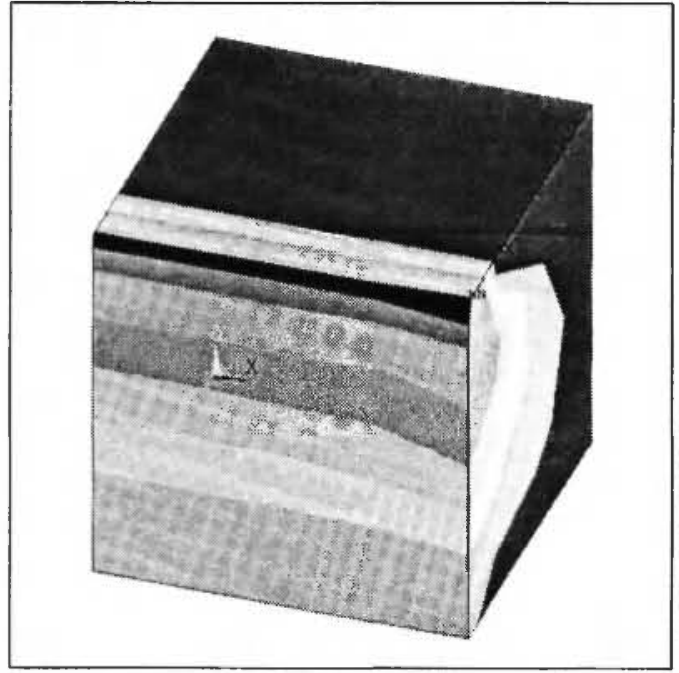

ANSYS 5.6

MAY $18 \quad 2000$

$13: 42: 37$

NODAL SOLUTION

STEP $=1$

TIME $=100$
SYZ

(AVG)

PowerGraphics

EFACET $=1$

AVRES=Mat

DMX $=.150897$

$\mathrm{SMN}=-62.382$

SMX $=2.521$

$-62.382$

$-55.17$

-47.959
$\square-40.748$

$-40.748$

$-26.325$

$-19.113$

$-11.902$

$-4.691$

2.521

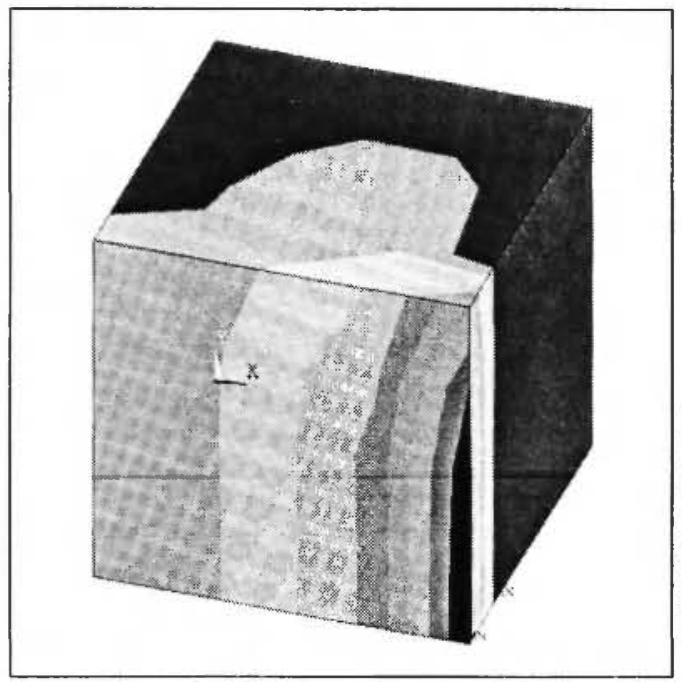

ANSYS 5.6

MAY $18 \quad 2000$

13:43:10

NODAL SOLUTION

STEP $=1$

SUB $=7$

TIME $=100$

SXZ

(AVG)

RSYS $=0$

PowerGraphics

EFACET $=1$

AVRE $S=$ Mat

$\mathrm{DMX}=.150897$

$S M N=-26.313$

SMX $=2.454$

$-26.313$

$-23.116$

$-19.92$

$-16.724$

$-13.527$

$-10.331$

$-7.135$

$-3.938$

$-.742129$

2.454

Fig. 9: Distribution of stress components $\sigma_{y}, \tau_{y z}$ and $\tau_{x z}$ inside unidirectional specimens when $f=0.3$ 
fiber direction, but the shear stress $\tau_{\underline{y z}}$ and transverse normal stress $\sigma_{y}$ in the middle of the edge perpendicular to the fiber direction. In two cases, the maximum magnitude of the shear stress $\tau_{y z}$ is approximately equal to the product of the coefficient of friction and the nominal TTH compressive stress $\sigma_{\mathrm{z}}$. This indicates that, according to Coulomb Law, relative slide takes place in the $y$ direction at the local regions of contact surfaces. However, the shear stress $\tau_{x z}$ is of smaller magnitude than the shear stress $\tau_{\mathrm{yz}}$, thereby indicating that no relative slide happens in the $\mathrm{x}$ direction at the contact surfaces. These characteristics are attributed to the large stiffness difference of unidirectional composites in the $\mathrm{x}$ and $\mathrm{y}$ directions. Under the TTH compressive stress, the free deformation of unidirectional specimens is much larger in the transverse (i.e., $y$ ) direction than in the fiber (i.e., $x$ ) direction due to the Poisson's effects. The isotropic collars thus impose strong constraints onto the unidirectional specimens through the friction force because of stiffness mismatch between them, especially in the $y$ direction. The maximum friction force the isotropic collars can impose is the product of the TTH compressive stress and the coefficient of friction at the critical moment when the slide is impending over the contact surfaces. Immediately prior to the specimen failure, the TTH compressive stress which is close to the TTH compressive strength always generate large deformation at the entire edges parallel to the fiber directions so as to make slide occur in the $y$ direction. Therefore, the maximum shear stress $\tau_{\mathrm{yz}}$ at the contact surface is proportional to the coefficient of friction, as displayed in Figures 8 and 9 . In the $x$ direction, however, the stiffness mismatch between the isotropic collars and unidirectional specimens is small, and thus no relative slide exists in the $\mathrm{x}$ direction at the edges perpendicular to the fiber directions. Consequently, the maximum shear stress $\tau_{x z}$ at the contact surface is always smaller than the product of the TTH compressive stress and the coefficient of friction. Moreover, because the free expansion of unidirectional specimens in the $y$ direction is constrained by the aluminum collars, a negative state for the stress $\sigma_{y}$ is generated near the contact surfaces inside specimens and attains maximum magnitude in the middle (i.e., $y=$ 0 ). Local free deformation in the $\mathrm{x}$ direction would result from the stress $\sigma_{y}$ inside the specimens due to the Poisson's effects, which is constrained by the collars. Therefore, the shear stress $\tau_{x z}$ is formed from the combined contributions of the Poisson's effects due to the TTH compressive stress $\sigma_{z}$ and the transverse normal stress $\sigma_{y}$. A maximum magnitude of the stress $\tau_{x z}$ exists at the middle of the edges perpendicular to the fiber direction, which results from the Poisson's effects due to the maximum state of the stress $\sigma_{y}$ at these locations.

Therefore, the coefficient of friction and the collar modulus play an important role in the determination of stress distribution inside the unidirectional specimens. In order to guarantee that the material failure takes place inside the specimen gage sections and to obtain uniform stress distribution inside the specimens, the coefficient of friction should be reduced or the collar materials with stiffness comparable to the unidirectional specimens in both the longitudinal and transverse should be employed. The first solution could be partially achieved by smearing some grease at the contact surfaces. The second solution, however, is difficult to achieve since the unidirectional specimens exhibit large stiffness difference in the longitudinal and transverse directions. On the other hand, the modulus of cross-ply specimens is of identical magnitude in the $\mathrm{x}$ and $\mathrm{y}$ directions, and thus the stiffness mismatch between the cross-ply specimens and steel collars is smaller in both the directions. Even so, the maximum shear stress is still significant at the contact surfaces between cross-ply specimens and steel collars immediately prior to the specimen failure because the TTH compressive strength of cross-ply specimens is much larger than that of unidirectional specimens. Hence, the most efficient approach towards determining the reliable TTH compressive strengths is to reduce the coefficient of friction at the contact surfaces between the specimens and collars.

It is noteworthy that the stress analysis is performed corresponding to an idealistic situation; i.e., the top and bottom surfaces are assumed to be parallel to each other. However, higher shear stress state would be generated, if these two surfaces were not parallel to each other. Therefore, it is important to examine the parallelism of specimens before testing; only the specimens with parallel surfaces are accepted. One of the most efficient 
approaches to prevent non-parallelism is to cut specimens far away from the edges of the thick laminates.

\section{SPECIMEN PREPARATION AND TESTING PROCEDURE}

The unidirectional and cross-ply laminates composed of 72 plies are fabricated from the G40800/5276-1 carbon/epoxy pre-impregnated tapes and cured in an autoclave following a specified curing cycle. Three thermal couples are inserted inside the laminates to monitor the temperature field through the laminate thickness in the curing process. The steel dams are used closely around the thick laminates to prevent the excess flowing-out of epoxy. Several bundles of glass fiber yarns are placed between the dams and the laminates and conducted to the breather, in order to form a channel to allow the entrapped air and bubble to escape out of the laminates.

The prism specimens are then cut from the laminated plates using a diamond wheel blade. Due to poor epoxy, the boundary regions of the laminated plates with a width of at least $25.4 \mathrm{~mm}$ are not used in order to guarantee the parallelism of specimens. The edges of the specimens are ground using sand-papers with high grit orders to remove the potential damage due to cutting. The top and bottom surfaces of specimens are lubricated by smearing some grease and the specimens are then mounted on a MTS testing machine. A compressive load is applied to the specimens by moving the plateau upward at a constant speed of $0.8 \mathrm{~mm} / \mathrm{min}$. and terminated when the load suddenly drops down to zero. During the loading process, the relative displacements and the corresponding loads are directly recorded from the MTS testing machine.

\section{EXPERIMENTAL RESULTS}

\subsection{Unidirectional Specimens}

The typical stress-strain curves for group 3 and group 4 specimens are shown in Figure 10. The strain is approximately determined by dividing the recorded movement of the testing machine, which represents the

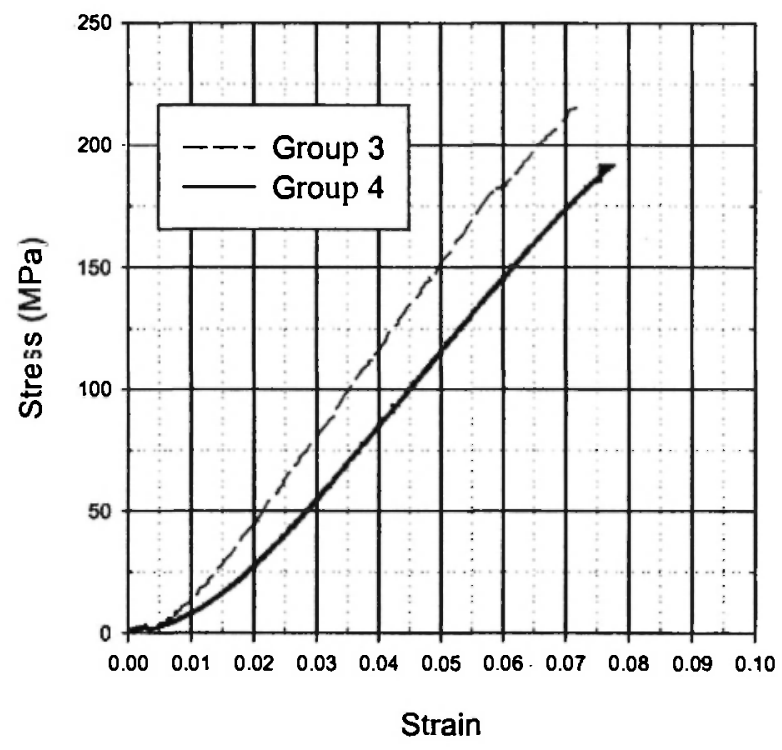

Fig. 10: Typical stress-strain curve for unidirectional specimens.

summation of the specimen deformation and the aluminum collar deformation, by the specimen thickness. The collar deformation, however, is much smaller than the specimen deformation, because the modulus of aluminum is much higher than the TTH modulus of graphite/epoxy composites. In spite of the collar deformation, it can be seen that unidirectional specimens exhibit a linear stress-strain behavior in the whole loading process. Likewise the stress-strain relationships are also observed from the other groups of specimens. The TTH compressive strength for each specimen is obtained by dividing the failure load by its cross-sectional area. The distribution of each individual sample value with respect to the corresponding specimen cross-sectional area is displayed in Figure 11, and the mean values $(\mu)$ and the coefficients of variations $(\mathrm{Cv})$ for the four groups of unidirectional specimens are listed in Table 3. The experimental data from group 1 specimens present smaller variation, but those from the remaining groups of specimens present greater variation.

A number of transverse cracks can be observed on the sides normal to the fiber directions in each failed specimen. The cracks grow in a tree-branch manner; secondary cracks are generated at the tips of primary cracks and their propagation directions deviate from 


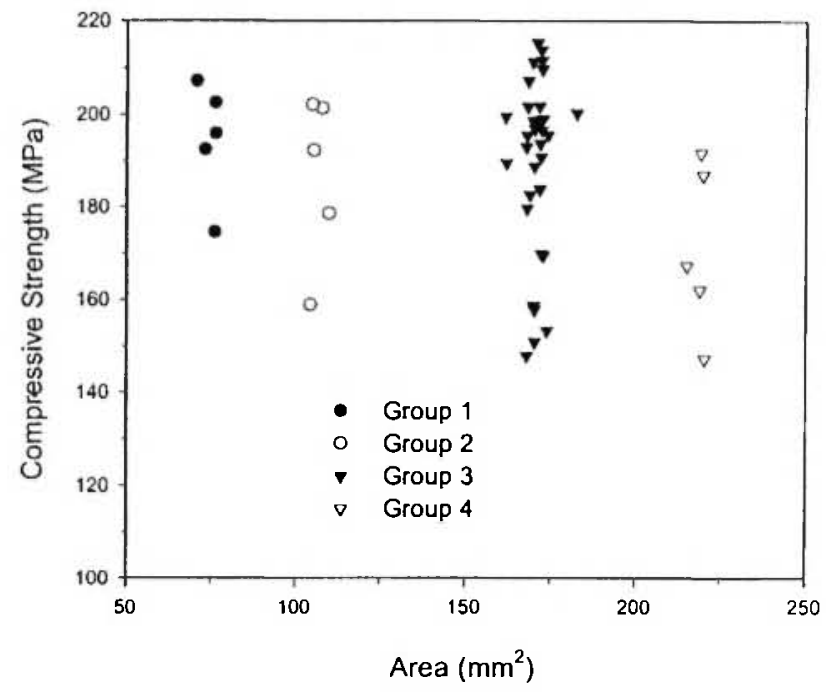

Fig. 11: Sample value distribution of TTH compressive strength $Z_{C}$ with respect to cross-section area obtained from unidirectional specimens.

Table 3

Experimental results of TTH compressive strengths for G40-800/5276-1 composite material.

\begin{tabular}{|c|c|c|c|c|c|}
\hline & \multicolumn{4}{|c|}{ Unidirectional } & Cross-Ply \\
\cline { 2 - 5 } & Group 1 & Group 2 & Group 3 & Group 4 & \\
\hline$\mu(\mathrm{MPa})$ & 194.53 & 186.62 & 189.59 & 171.10 & 1008.88 \\
\hline $\mathrm{Cv}(\%)$ & 6.44 & 9.73 & 10.10 & 10.71 & 5.26 \\
\hline
\end{tabular}

those of the primary cracks. Consequently, the transverse cracks are found to propagate to the two side surfaces parallel to the fiber directions. Therefore, the transverse cracks are generated in the whole gage section of specimens. This evidence shows that the transverse cracks grow easily in unidirectional prism specimens, because the fibers make no contributions to arresting the transverse crack propagation. Hence, the failure mechanism of the G40-800/5276-1 unidirectional composite material in the TTH compressive test is different from those reported by Goeke $/ 7 /$ for woven and knit-stiched materials. Figure 12 displays the side view of matrix cracks with respect to one of the group 3 specimens. The aforementioned fracture mode happens in all the groups of specimens.

From the failure mode, an interesting proof can also be obtained that the transverse cracks propagate grossly

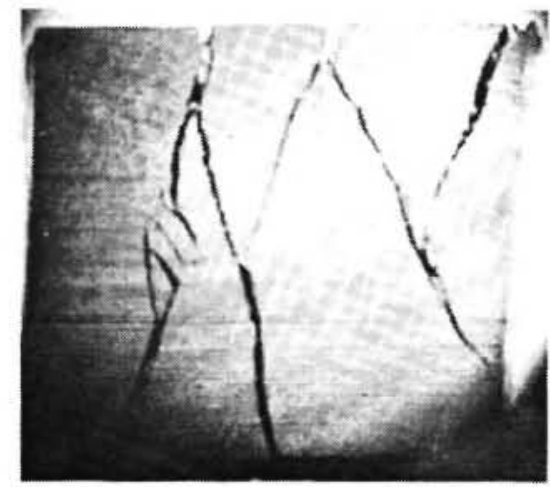

Fig. 12: Side view (from the fiber direction) of matrix cracks in unidirectional specimens.

along a direction of $\theta=10^{\circ}$ with respect to the compressive loading direction (i.e., the TTH direction). The TTH compressive normal stress $\sigma_{z}$ can be related to the shear stress $\tau$ along the crack surfaces by

$$
\sigma_{z}=\frac{\tau}{\sin (2 \theta)}
$$

If one substitutes the transverse interlaminar shear strength $S_{23}$ for $\tau$ in the above equation, one can find that the normal stress $\sigma_{z}$ would be very close to the TTH compressive strength. This could indicate that the transverse matrix cracks are formed when the TTH compressive load is increased to a point where the shear stress $\tau$ at the crack surfaces reaches the transverse interlaminar shear strength of the material. Therefore, it could be concluded that the TTH compressive failure of unidirectional specimens is governed by the transverse matrix cracks due to shear. It is noteworthy that unidirectional composites are transversely isotropic and thus the transverse interlaminar shear strength $S_{23}$ can be substituted for $\tau$ along the crack surfaces in Equation (3).

\subsection{Cross-Ply Specimens}

The typical stress-strain curve for the cross-ply specimens is shown in Figure 13, wherein the strain is approximately determined by dividing the summation of the specimen deformation and carbon steel collar deformation by the specimen thickness. Similarly, 
because the modulus of carbon steel is much higher than the TTH modulus of specimens, the contribution of the deformation from carbon steel collars to the TTH strain is negligible. Therefore, it is accurate that the displayed strain in Figure 13 is considered to be the TTH strain of specimens. A linear stress-strain behavior is also demonstrated until the peak of the whole loading process. Larger TTH deformation is presented by the cross-ply specimens than by the unidirectional specimens before the final failure. Comparing the slope of the stress-strain curves in Figures 10 and 13, it is found that the cross-ply specimens have a TTH modulus of $5.7 \mathrm{GPa}$ which is larger than that of the unidirectional specimens (i.e., 3.2 GPa). Therefore, a conclusion could be made that the fiber orientations affect the TTH modulus to some extent.

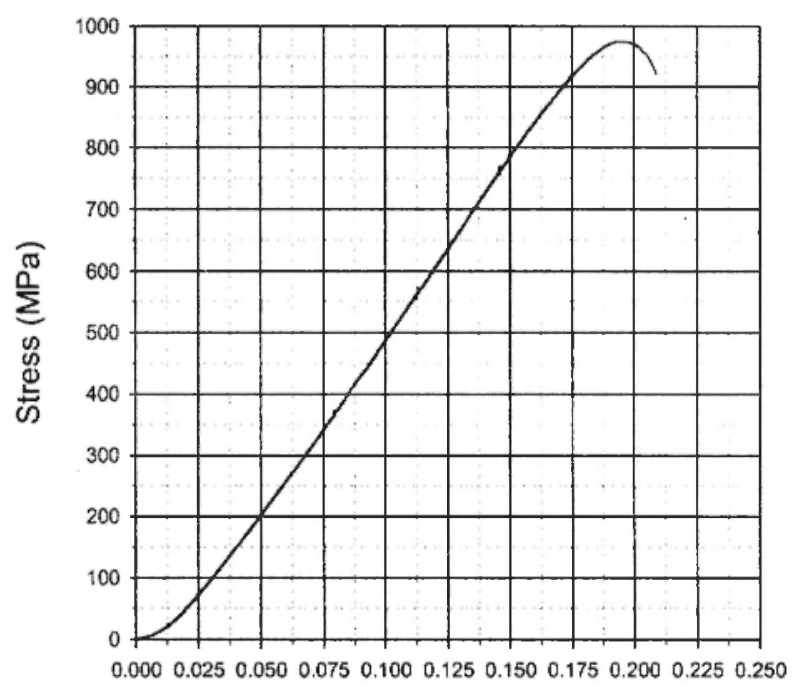

Strain

Fig. 13: Typical stress-strain curve for cross-ply specimens.

The mean value and the coefficient of variation of the TTH compressive strength are also presented in Table 3. The TTH compressive strength of cross-ply specimens is much higher than that of unidirectional specimens, and further has a much smaller coefficient of variation. The unidirectional specimens are sensitive to the matrix defects introduced in the manufacturing process; the concentrated stress due to matrix defects quickly makes the formation of macro matrix-cracks through the laminate thickness, since all the fibers in the unidirectional specimens are oriented in the same direction. The fracture surfaces of matrix defects, or the fracture surfaces of micro-cracks initiated from the matrix defects at the initial loading stage, are always parallel to the fiber direction. Along some surfaces which are slightly inclined to the loading direction, the concentrated shear stresses are large enough to make these initial matrix cracks propagate along a plane. Therefore, the planar crack surfaces are always parallel to the fibers and slightly inclined to the loading direction for unidirectional specimens, as shown in Figure 12. This indicates that the fibers in unidirectional specimens could not arrest the crack growth. Hence, the TTH compressive strengths from the unidirectional specimens exhibit lower value and higher scatter. In the cross-ply specimens, however, the fibers in each ply are perpendicular to the fibers in its two surrounding plies. Consequently, the fracture surfaces of matrix defects, or the fracture surfaces of initial micro-cracks in one ply, are perpendicular to the fibers of the two surrounding plies, which therefore prevent these initial matrix cracks from quickly growing through the laminate thickness. The initial matrix cracks will thus grow in two possible directions; going forward through the neighboring plies or along the fiber directions. In the first case, the fibers will be broken in order to allow the cracks going to the neighboring plies, thereby requiring high external load. In the second case, the directions of the initial matrix cracks have to be changed and propagate in a planar way parallel to the interfaces. When subjected to the TTH compressive loading, only the nominal TTH compressive stress component is of large magnitude at the interfaces, which does not contribute much to the planar failure parallel to the interfaces. The other stress components that play a dominant role in the planar failure, such as $\tau_{x z}$ and $\tau_{y z}$, are indirectly generated due to the matrix defects through deformation compatibility. Consequently, the external load must be very high in order that these stress components are large enough to form the macro-cracks. Therefore, compared with unidirectional specimens, the cross-ply specimens are not so sensitive to the matrix defects, and present higher TTH compressive strength and lower scatter. The previous explanations can be verified by observing the fracture surfaces of cross-ply specimens, which are 
displayed in Figure 14. The fracture surfaces of crossply specimens are not flat; accompanied by crack jumping, a number of local planar cracks parallel to the interfaces are generated at different heights in the thickness directions. The crack jumping between local planar cracks is formed through some local TTH cracks, the fracture surfaces of which are roughly normal to the interfaces. Therefore, considerable fiber breakage can be observed at the fracture surfaces of local TTH cracks, which is attributed to the highly concentrated stress in the fiber directions at the crack tips when the cracks are growing forward.

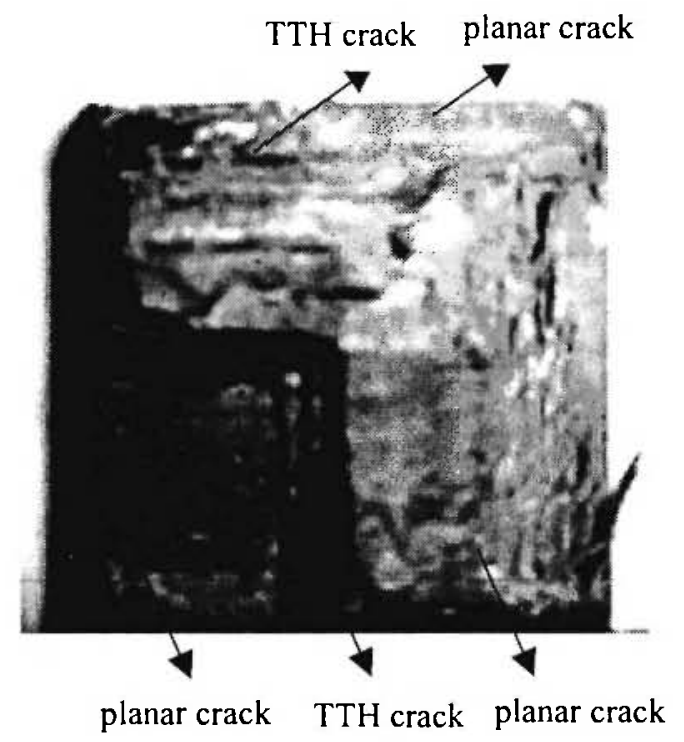

Fig. 14: Front view (from the TTH direction) of fracture surfaces in cross-ply specimens.

\section{STATISTICAL ANALYSIS}

Four batches of sample values have been obtained from four types of prism specimens with different sizes. The statistical analysis will be performed to determine whether the four batches of sample values come from the same population. If so, a conclusion could be made that there would be no size effects on the TTH compressive strength of unidirectional laminates, and the four batches could be combined together to derive the probability distribution function for the TTH compressive strength of the G40-800/5276-1 unidirectional laminates.
The rank-sum test technique is usually employed to judge whether two batches of samples come from the same population $/ 11 \%$. Suppose that $X_{i}(i=1, \ldots, n)$, and $Y_{j}(j=1, \ldots, m)$ are two batches of sample values that are independently obtained. These two batches of sample values are combined together and ranked in an ascending sequence. The sum of the ranks with respect to $X_{i}$ and $Y_{j}$ in the combination can thus be calculated, which are hereby designated as $W_{X}$ and $W_{Y}$ respectively. Then, it can be concluded that $X_{1}$ and $Y_{j}$ come from the same population with a given confidence $(1-\alpha) \%$, provided that $t_{\alpha / 2}<W_{Y}<t_{1-\alpha / 2}$; the confidence limits are defined as $/ 11 /$

$$
\begin{aligned}
& t_{\alpha / 2}=\frac{m(m+n+1)}{2}+z_{\alpha / 2} \sqrt{m n(m+n+1) / 12}-\frac{1}{2} \\
& t_{1-\alpha / 2} \cong \frac{m(m+n+1)}{2}+z_{1-\alpha / 2} \sqrt{m n(m+n+1) / 12}+\frac{1}{2}
\end{aligned}
$$

and $\mathrm{N}\left(\mathrm{z}_{\alpha / 2}\right)=\alpha / 2, \mathrm{~N}\left(\mathrm{z}_{1-\alpha / 2}\right)=1-\alpha / 2$ (here $\mathrm{N}$ denotes the standard normal distribution). Using the rank-sum test technique, one can make a judgement on the so-called size effects; i.e., whether the four batches of TTH compressive strength values form unidirectional specimens with different sizes come from the same population at a given confidence. In a physical sense, the rank-sums within the confidence limits indicate that the two batches of sample values are thoroughly intermixed, thereby being viewed as sample values of a single random variable.

The test procedures are described as follows: (a) Combine group 1 and other three groups and sort out the sample values; (b) Sum the ranks for the two groups of sample values in each combination; (c) Compare the ranks with the confidence limits calculated from Equations (4) and (5). In the following, given $\alpha=0.05$, the rank-sum test is performed on the three combinations of TTH compressive strength values. The sorted sample values, the rank-sums for the three combinations and the calculated confidence limits are tabulated in Table 4. The rank-sums are found to be within the confidence limits for combinations 1 and 2, but out of the confidence limit for combination 3 . Hence, it can be concluded that TTH compressive strength values of groups 1, 2 and 3 represent the 
Table 4

Rank-sum test results of the sample values from different groups

\begin{tabular}{|c|c|c|c|c|}
\hline Older & Group I & Group 2 & Group 3 & Group 4 \\
\hline 1 & 174.59 & 158.88 & 148.03 & 147.28 \\
\hline 2 & 192.48 & 178.61 & 150.97 & 162.17 \\
\hline 3 & 195.91 & 192.27 & 153.44 & 167.27 \\
\hline 4 & 202.50 & 201.30 & 157.98 & 186.96 \\
\hline 5 & 207.16 & 202.04 & 158.73 & 191.85 \\
\hline 6 & & & 169.65 & \\
\hline 7 & & & 169.95 & \\
\hline 8 & & & 179.83 & \\
\hline 9 & & & 182.79 & \\
\hline 10 & & & 183.96 & \\
\hline 11 & & & 188.91 & \\
\hline 12 & & & 189.72 & \\
\hline 13 & & & 190.92 & \\
\hline 14 & & & 193.19 & \\
\hline 15 & & & 193.85 & \\
\hline 16 & & & 195.62 & \\
\hline 17 & & & 195.72 & \\
\hline 18 & & & 196.68 & \\
\hline 19 & & & 197.00 & \\
\hline 20 & & & 198.59 & \\
\hline 21 & & & 198.95 & \\
\hline 22 & & & 199.14 & \\
\hline 23 & & & 199.70 & \\
\hline 24 & & & 200.43 & \\
\hline 25 & & & 201.83 & \\
\hline 26 & & & 201.87 & \\
\hline 27 & & & 207.34 & \\
\hline 28 & & & 209.79 & \\
\hline 29 & & & 211.40 & \\
\hline 30 & & & 211.65 & \\
\hline 31 & & & 213.87 & \\
\hline 32 & & & 215.42 & \\
\hline $\begin{array}{l}\text { Combination } \\
\text { Gr. } 1 \& \text { Gr. } 2\end{array}$ & \multicolumn{4}{|c|}{$\begin{array}{l}\mathrm{W}_{1}=32, \mathrm{~W}_{2}=23 \\
\mathrm{t}_{0.025}=17.6, \mathrm{t}_{0.975}=37.4 \\
\text { Therefore } \mathrm{t}_{0.025}<\mathrm{W}_{2}<\mathrm{t}_{0.975}\end{array}$} \\
\hline $\begin{array}{l}\text { Combination } \\
\text { Gr. } 1 \& \text { Gr. } 3\end{array}$ & \multicolumn{4}{|c|}{$\begin{array}{l}W_{1}=104, W_{3}=599 \\
t_{0.025}=563.4, t_{0.975}=652.6 \\
\text { Therefore } t_{0.025}<W_{3}<t_{0.975}\end{array}$} \\
\hline $\begin{array}{l}\text { Combination } \\
\text { Gr. } 1 \& \text { Gr. } 4\end{array}$ & \multicolumn{4}{|c|}{$\begin{array}{l}W_{1}=38, W_{4}=17 \\
t_{0.025}=17.6, t_{0.975}=37.4 \\
\text { Therefore } W_{4} \text { is out of range }\left[t_{0.025}, t_{0.975}\right]\end{array}$} \\
\hline
\end{tabular}

sample values coming froin the same population at a 95\% confidence level, and thus the TTH compressive strength values in the first three groups can be represented by one probability function. However, the TTH compressive strength values of group 4 cannot be considered to come from the same population as that of group 1 at a $95 \%$ confidence level, thereby having a different probability function from that for the sample values of groups 1,2 and 3.

Therefore, the TTH compressive strength values of groups 1, 2, and 3 are combined together and then their probability distribution is estimated using the twoparameter Weibull function described in Equation (2). The theoretical probability distribution and the sample probability distribution obtained from Equation (2) are displayed in Figure 15. The mean value of the TTH compressive strength is $189.83 \mathrm{MPa}$ and the coefficient of variation is $9.55 \%$ after the combination. The goodness-of-fit between the theoretical and sample probability distributions is evaluated by the Kolmogorov-Smirnov test technique $/ 11 \%$. That is, the theoretical probability distributions are accepted, if the maximum difference, Dn, between the theoretical and sample probability distributions does not exceed the associated acceptance limits. The acceptance limits are equal to $1.36 / \sqrt{n}$ at $95 \%$ confidence level. For the TTH compressive strength values, the Kolmogorov-Smirnov statistic Dn is found to be 0.097 , which is much smaller than the acceptance limit 0.209 with respect to $n=42$. This indicates that the TTH compressive sample values can be well fitted by the two-parameter Weibull distribution. By comparing the shape parameters, one can find that the TTH compressive strength has the

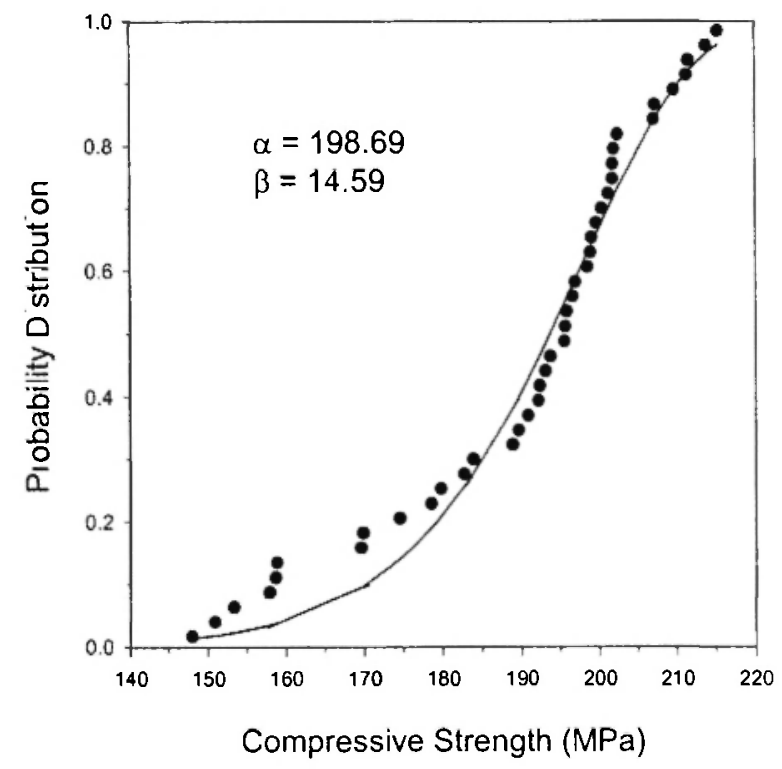

Fig. 15: Probability distribution of TTH compressive strength $Z_{C}$ obtained from unidirectional specimens. 
same degree of scatter as the transverse interlaminar shear strength and these two strength components pose smaller variation in the matrix-dominated strengths. On the basis of the rank-sum test results, a conclusion can be made that the TTH compressive strength values from the unidirectional specimens present size effects to some degree. However, the inherent size effects do not seem to be apparent, because the strength values within groups 1, 2 and 3 seem to be independent of specimen sizes. Nevertheless, the investigation on size effects is not sufficient in the present paper, because the ranges of specimen sizes tested are narrow. The size effects on the TTH compressive strength of laminated composites should be investigated through testing a sufficiently wide range of specimen sizes.

\section{CONCLUSIONS}

The TTH compressive test based on the end-loaded prism specimens has been demonstrated to be an effective test technique towards the TTH compressive strengths of laminated composites in the present experimental program. The coefficient of friction between the prism specimens and the collars is an important factor that will affect the experimental results, and thus should be reduced as low as possible.

The experimental results show that the TTH compressive strength of laminated composites depends on the lay-ups; the value for cross-ply specimens is roughly five times as much as that for unidirectional specimens, and so does the TTH compressive modulus. Also, the TTH compressive strength of unidirectional specimens presents size effects to some degree. Compared with the other matrix-dominated strengths, the TTH compressive strength demonstrates smaller scatter, and the inherent randomness can well be represented by the two-parameter Weibull probability functions.

\section{ACKNOWLEDGEMENT}

The support of the Natural Sciences and Engineering Research Council of Canada (NSERC) through a grant (grant \# 418-98) is gratefully appreciated. C. Zhang would also like to thank the NSERC and the Canadian Space Agency Committee for the postgraduate scholarships.

\section{REFERENCES}

1. S.W. Tsai and E.M. Wu, "A general theory of strength for anisotropic materials," Journal of Composite Materials, 5, 58-80 (1971).

2. O. Hoffman, "The brittle strength of orthotropic materials," Journal of Composite Materials, 1, 200206 (1967).

3. P.A. Lagace and D.B. Weems, "A through-thethickness strength specimen for composites," Test Methods for Design Allowables for Fibrous Composites, ASTM STP 1003, 1989, pp. 197-207.

4. C.C. Hiel, M. Sumich and D.P. Chappell, "A curved beam test specimen for determining the interlaminar tensile strength of laminated composites," Journal of Composite Materials, 25, 854-868 (1991).

5. W.C. Jackson and R.H. Martin, "An interlaminar tensile strength specimen," Composite Materials: Testing and Design, ASTM STP, 1206, 1993, pp. 333-354.

6. W.C. Jackson, "Through-the-thickness test strength of textile composites," Composite Materials, Testing and Design, ASTM STP 1274, 1996, pp. 218-238.

7. E.G. Goeke, "Compression of "thick" composites: two test methods", Composite Materials: Testing and Design, ASTM STP, 1206, 1993, pp. 90-102.

8. M.L. Benzeggagh, K. Khellil and T. Chotard, "Experimental determination of Tsai failure tensorial terms Fij for unidirectional composite materials," Composites Science and Technology, 55, 145-156 (1995).

9. C. Zhang, "Random delamination of graphite/epoxy laminated composites: stress analysis, failure criteria, experimental characterization and stochastic modeling," Ph.D. Dissertation, Concordia University, Montreal, Canada, 2001.

10. C. Zhang, S.V. Hoa and R. Ganesan, "Experimental characterization of interlaminar shear strengths of graphite/epoxy laminated composites," Submitted to Journal of Composite Materials, 2000.

11. H.J. Larson, Introduction to Probability Theory and Statistical Interface, Third Edition, John Wiley and Sons, New York, 1982. 